\title{
A h-BCN for Electrochemical Sensor of Dopamine and Uric Acid
}

\author{
Yun-Shuai Bi, Bo Liu, Xu-Ying Liu, Ying Qin, and Ben-Xue Zou \\ Department of Chemical Engineering, Eastern Liaoning University, Dandong 118001, China \\ Correspondence should be addressed to Ben-Xue Zou; benxue_dd@163.com
}

Received 17 September 2019; Accepted 26 December 2019; Published 16 January 2020

Academic Editor: Yoke K. Yap

Copyright (c) 2020 Yun-Shuai Bi et al. This is an open access article distributed under the Creative Commons Attribution License, which permits unrestricted use, distribution, and reproduction in any medium, provided the original work is properly cited.

\begin{abstract}
A hexagonal boron carbon nitride hybrid $(\mathrm{h}-\mathrm{BCN})$ is developed by in situ high-temperature solid-state reaction and subsequent chemical reduction with hydrazine. The XRD and TEM results show that the h-BCN features interlayered structures with two characteristic $d$-spacing of 0.33 and $0.21 \mathrm{~nm}$. The obtained h-BCN exhibits significant electrochemical sensor for dopamine and uric acid. The cyclic voltammetric and amperometric experiments revealed a good linear relationship between current densities and concentrations of dopamine (DA) of $10-300 \mu \mathrm{M}$ and uric acid (UA) of 10-500 $\mu \mathrm{M}$, with high sensitivities of $0.14 \mu \mathrm{A} / \mu \mathrm{M}$ and $0.32 \mu \mathrm{A} / \mu \mathrm{M}$ and detection limits of $5 \mu \mathrm{M}$ and $2 \mu \mathrm{M}$, respectively.
\end{abstract}

\section{Introduction}

Graphene, a two-dimensional monolayer of carbon atom arranged in honeycomb network, has attracted tremendous attention due to its unique properties such as novel electronic properties, excellent mechanical flexibility, large surface area, and high thermal conductivity and chemical stability [1-4]. An infinite pristine graphene is semimetal with zero gap because its conduction and valence bands connect each other near the Dirac points. Hexagonal boron nitride (h-BN), known as "white graphite," is generally considered to be an electrically insulator with a wide bandgap of $5.97 \mathrm{eV}[5,6]$. The $2 \mathrm{D}$ structure of $\mathrm{h}-\mathrm{BN}$ is very similar to the graphene layer bonding with two different chemical species of boron and nitrogen atoms. It displays excellent thermal conductivity, good mechanical properties, and excellent chemical stability [7-10]. It had been widely used as solid lubricant in cosmetics, thermal conductivity filler in polymer, and additive materials in the ceramics and aerospace field. It is worth noted that the monolayer $\mathrm{h}-\mathrm{BN}$ behaves semiconducting nature theoretically and experimentally based on the fact that electron tunneling through few-layer h-BN along the $c$-axis and injection of mobile charge carriers at high field in $2 \mathrm{D}$ crystal $[11,12]$.

Hybridization of h-BN with graphene layers may effectively monitor the bandgap of h-BN or open up of graphene bandgap, which gives rise to a new novel class of semicon- ductor material of hybrid boron carbon nitride $(\mathrm{BCN})$ superlattic interlayered structures [13-16]. It has been demonstrated that semiconductor materials are most likely to form an electric double layers at the interface between the solid-state electrode and electrolyte solution. The interfacial electron transfer across semiconductor-liquid interfaces plays an important role in photoelectrocatalysis and electrocatalysis. The structural defects and surface state of the semiconductor electrode may have a dramatic effect on the mechanism of electron transfer. The different electronegativities of $\mathrm{B}, \mathrm{C}$, and $\mathrm{N}$ atoms result in desirable charge transfer and electronic properties in different kinds of $\mathrm{BCN}$ structures. The slightly larger B-N bond length (1.7\%) than that of $\mathrm{C}-\mathrm{C}$ bond makes it possible for alloying the h-BN with $\mathrm{C}$ atom with minimal internal stress [17].

Hybridized 2D h-BCN has two main structures. One kind is an alloyed $\mathrm{BCN}$ by substitution of $\mathrm{C}$ in graphene by $\mathrm{B}$ and $\mathrm{N}[18,19]$. The other is alloying $\mathrm{BN}$ and graphene in the phase-segregated $\mathrm{BCN}$ in which $\mathrm{BN}$ (graphene) retains its own phase and separated by graphene (BN) [20-22]. Depending on the growth process, different types of alloying are possible. Thus, preparation and characterization of h$\mathrm{BCN}$ semiconductor has been of increasing interest in recent years. $\mathrm{Ci}$ et al. used the thermal catalytic CVD growth method with $\mathrm{NH}_{3}-\mathrm{BH}_{3}$ as precursors to obtain large area of 2D h-BCN films on Cu substrate [23]. They found that a wide range of compositions may be engineered to build new 
semiconducting 2D architectures, which enables the development of bandgap-engineered applications in electronics and optics and properties that are distinct from those of graphene and h-BN. Chegel investigated the nature of $\mathrm{BN}$ and $\mathrm{BN} /$ graphene bilayers by using the tight binding approximation and Green function method. It had been revealed that $\mathrm{BN} /$ graphene bilayers have smaller gap and larger electrical conductivity than that of $\mathrm{BN}$ bilayers [24]. Saha et al. reported SAA functioned GO/h-BN superlattice electrode with high specific capacitance of $\sim 1300 \mathrm{Fg}^{-1}$ for supercapacitor applications [25]. F-doped $\mathrm{h}-\mathrm{BN} / \mathrm{rGO}$ superlattice through the transition from $\mathrm{n}$ - to p-type semiconductor with the specific capacitance of $1250 \mathrm{Fg}^{-1}$ for supercapacitors was also been explored by them [26]. They proposed that the $\mathrm{h}-\mathrm{BN} / \mathrm{rGO}$ superlattice behaved like n-type semiconductor due to the enriched electron density at the conduction band. In this work, a facial method was presented for preparation of layered $\mathrm{h}-\mathrm{BCN}$ and corresponding structures and electrochemical sensor properties for DA and UA were studied. The proposed method possesses mild reaction condition without using ammoniums gas and low cost. The constructed h-BCN electrode exhibits excellent sensor properties for electrochemical detection of dopamine and uric acid suggesting a good prospect for practical applications.

\section{Experimental Section}

2.1. Synthesis of $h-B C N$. The hexagonal boron carbon nitride (h-BCN) was prepared with anhydrous borax, melamine, and the low-molecular weight phenolic resin as starting materials. The low-molecular weight phenolic resin was prepared as our previous reports, followed by freeze drying for $2 \mathrm{~h}$ to avoid the agglomeration. Anhydrous borax, melamine, and the low molecular weight phenolic resin were milled with a weight ratio of $1: 1: 0.5$ using ball mill for $2 \mathrm{~h}$ at a rotation speed of $500 \mathrm{rpm}$. The mixed solid was heated in the air at $300^{\circ} \mathrm{C}$ for 2 hours. Then, the sample was calcinated at a tube furnace at $700^{\circ} \mathrm{C}$ for $1 \mathrm{~h}, 800^{\circ} \mathrm{C}$ for $1 \mathrm{~h}$, and $900^{\circ} \mathrm{C}$ for $2 \mathrm{~h}$ under pure $\mathrm{N}_{2}$ atmosphere, through temperature-programmed route with a heating rate of $10^{\circ} \mathrm{C} / \mathrm{min}$. The $\mathrm{N}_{2}$ flow rate was adjusted $5 \sim 10 \mathrm{ml} / \mathrm{min}$. The as-made composite was then placed in a hydrazine solution to reduce oxygen functional group, followed by filtration, drying in the air, and finally ball mill mixing for $2 \mathrm{~h}$. The obtained powder was donated as h-BCN.

2.2. Synthesis of $h-B N$. Typically, anhydrous borax and melamine with a weight ratio of 10:7 was first mixed with ball mill for $2 \mathrm{~h}$. The mixed solid was heated in the air at $300^{\circ} \mathrm{C}$ for 2 hours and then calcinated at a tube furnace at $700^{\circ} \mathrm{C}$ for $1 \mathrm{~h}, 800^{\circ} \mathrm{C}$ for $1 \mathrm{~h}$, and $900^{\circ} \mathrm{C}$ for $2 \mathrm{~h}$ under pure nitrogen atmosphere, through temperatureprogrammed route with a heating rate of $10^{\circ} \mathrm{C} / \mathrm{min}$. The obtained solid was washed with water to remove the residual boron oxide and filtered through a polytetrafluoroethylene membrane, and the resulting solids were washed with water for several times, followed by drying

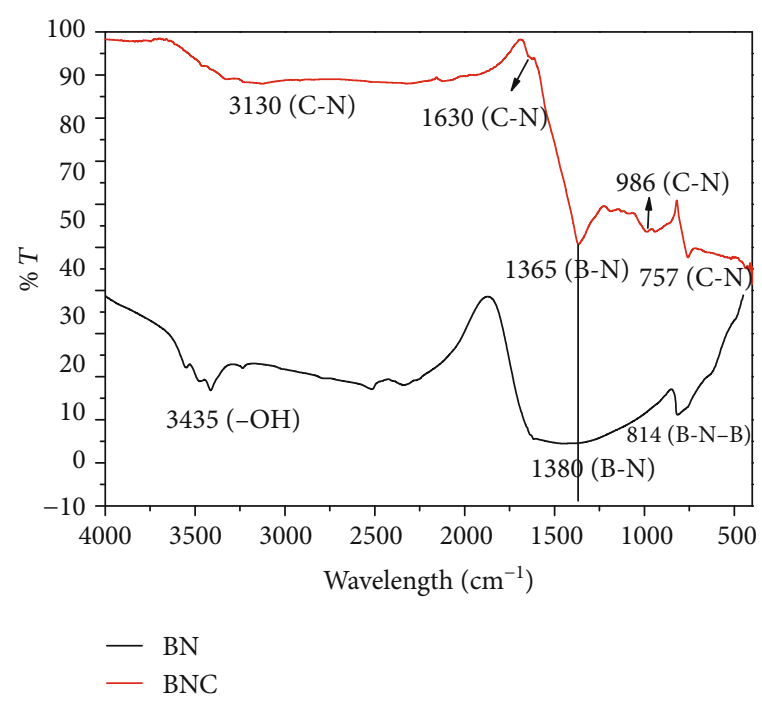

FIgURE 1: Infrared spectra of h-BCN and h-BN samples.

at $50^{\circ} \mathrm{C}$ in vacuum for $12 \mathrm{~h}$ and ball mill mixing for $2 \mathrm{~h}$. The obtained powder was donated as h-BN.

2.3. Materials Characterization. Fourier-transform infrared (FTIR) spectra were recorded using a Nicolet Nexus 470 FTIR spectrophotometer (Thermo Nicolet, USA). The morphologies of the samples were investigated by field-emission scanning electron microscopy (FESEM, Carl Zeiss, Germany) and high-resolution transmission electron microscopy (HRTEM, JEM2010-HR, $200 \mathrm{kV}$ ). X-ray diffraction measurement (XRD) was conducted to determine the crystal structures of the samples. XPS spectra were recorded on Thermo ECSALAB 250 electron spectrometer using $\mathrm{Al} \mathrm{K \alpha}$ radiation $(1486.6 \mathrm{eV})$. The $\mathrm{UV}$-vis spectra were recorded on an Analytik Jena SPECORD S600 UV-vis spectrometer (Germany).

2.4. Electrochemical Measurement. All the electrochemical experiments were performed on CHI 660E potentiostat (China) in a conventional three-electrode system with a platinum wire as the counter electrode and saturated calomel electrode (SCE) as the reference electrode. Carbon cloth was used as the working electrode's current collector. Before used, the carbon cloth was cleaned by acetone and water several times. The h-BCN-modified carbon cloth electrodes (h$\mathrm{BCN} /$ carbon cloth) were prepared by pasting the asprepared $\mathrm{h}-\mathrm{BCN}$ powders with an inactive carbon colloid binder on the carbon cloth. The cyclic voltammograms (CVs) and amperometric were recorded in deoxygenated phosphate-buffered solution ( $\mathrm{pH}$ 6.8) at room temperature. The $\mathrm{CV}$ was performed from -0.2 to $0.8 \mathrm{~V}$ with a scan rate of $10 \mathrm{mV} / \mathrm{s}$.

\section{Results and Discussion}

3.1. Characterization of $h-B C N$. Figure 1 shows the FTIR spectra of the obtained h-BNC and h-BN. The strong band at $1365 \mathrm{~cm}^{-1}$ is attributed to the characteristic stretching 


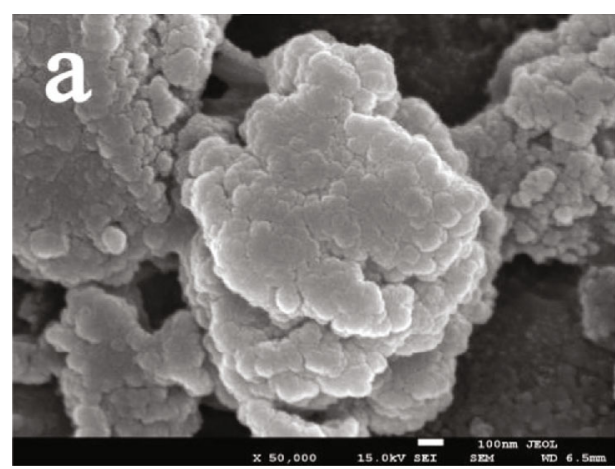

(a)

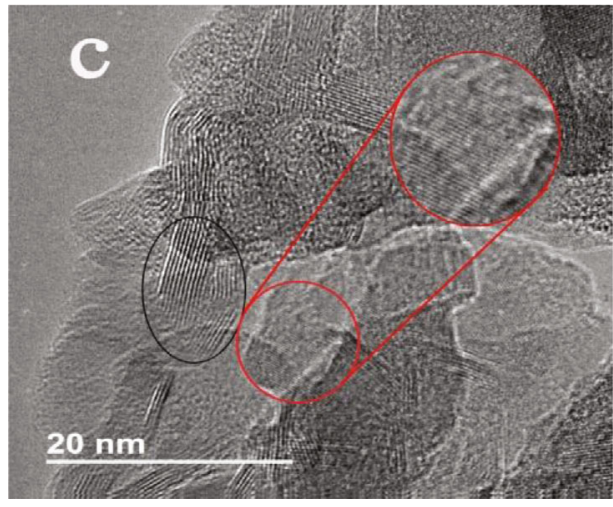

(c)

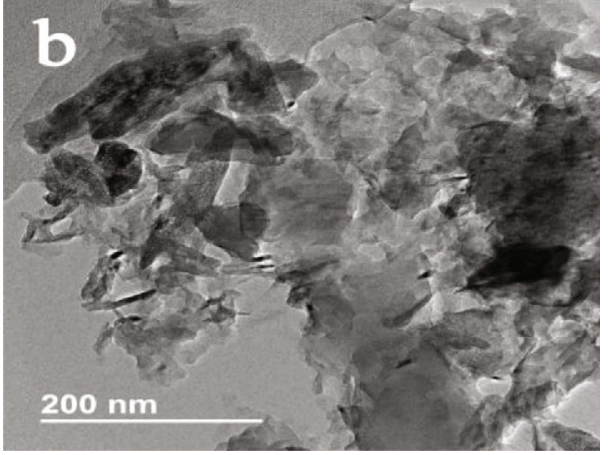

(b)

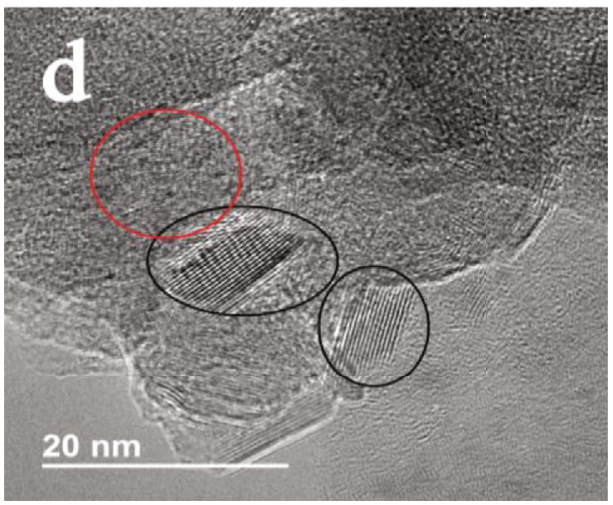

(d)

FIGURE 2: (a) Low resolution of SEM for h-BCN. (b, c, and d) High resolution of TEM for h-BCN sample; the larger red inset in (c) shows the expansion of image in the small red circle.

mode of $\mathrm{B}-\mathrm{N}[14,15]$. The band at $3130 \mathrm{~cm}^{-1}$ is assigned to be the stretching mode of the $\mathrm{C}-\mathrm{H}$ group [7]. The bands appeared at 1630,986 , and $757 \mathrm{~cm}^{-1}$ are attributed to the stretching of the C-N group $[27,28]$. The prepared h-BNC sample has the same mode of the B-N-B group with the BN sample. These results show that the $\mathrm{B}-\mathrm{N}$ and $\mathrm{C}-\mathrm{N}$ groups presented in the prepared h-BNC sample.

The morphology of the h-BNC sample was inspected by FESEM and TEM. SEM images shown in Figure 2(a) presented numbers of blocks aggregated by several platelet structures with average diameter of about $2 \mu \mathrm{M}$. The low magnification TEM images, as shown in Figure 2(b), of the $\mathrm{h}-\mathrm{BCN}$ are transparent to an electron beam due to their thin layered shapes. High-resolution TEM imaging further revealed thin graphene-liked multilayer sheets or lamellar structures of h-BCN (Figures 2(c) and 2(d)). The two different interlayer lattice fringes of $0.34 \mathrm{~nm}$ and $0.21 \mathrm{~nm}$ were clearly observed within the h-BCN, indicating the h-BCN layer is well crystallized. The lattice fringes of $0.34 \mathrm{~nm}$ (marked in black circles) and $0.2 \mathrm{~nm}$ (red circles) should be associated with the $d$-spacing of (002) and (100) planes in the BN-like or graphite-like crystal structure [26]. TEM images also exhibit thin disordered and open edges consisting of interlinked layer structures. The large number of these exposed edges of planes can be regarded as reactive sites for the enhanced electrochemical behaviors.

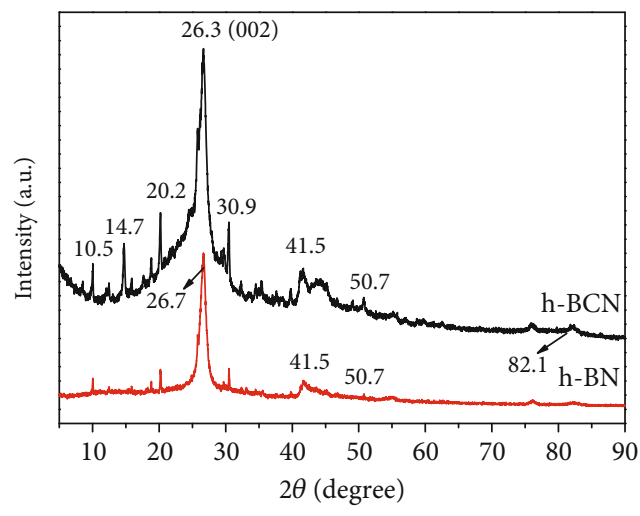

FIgURE 3: X-ray diffraction patterns of h-BCN and h-BN.

The XRD patterns of the $\mathrm{h}-\mathrm{BCN}$ sample showed characteristic diffraction peaks of hexagonal BNC (JCPDS no. 00052-0233) in Figure 3. The diffraction peaks at $26.3^{\circ}$ and $41.1^{\circ}$ correspond to (002) and (100) lattice planes within hBCN crystal structures [27-29]. The lattice $d$-spacing of (002) and (100) plane is about 0.34 and $0.21 \mathrm{~nm}$, respectively, which is consistent with the observations from TEM results. The diffraction peaks appeared at $14.7^{\circ}, 26.3^{\circ}, 41.5^{\circ}, 50.7^{\circ}$, and $82.1^{\circ}$ can be indexed to the planes of hexagonal BN (JCPDS no. 01-074-1978). The diffraction peaks at $26.3^{\circ}$, $41.5^{\circ}, 50.7^{\circ}$, and $82.1^{\circ}$ can be assigned to the planes of 


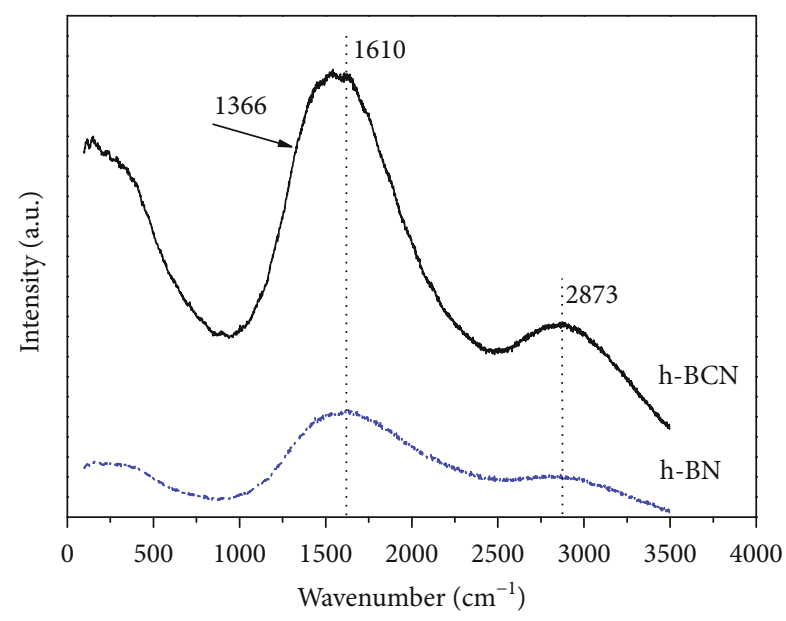

Figure 4: Raman spectra of h-BCN and h-BN.

hexagonal graphite C (JCPDS no. 00-023-0064). These remarkable diffraction peaks for h-BCN sample are similar to the characteristic diffraction peaks of hexagonal graphite carbon and $\mathrm{h}-\mathrm{BN}$, indicating that the obtained $\mathrm{h}$-BNC possess well-hybridized structures of h-BN and graphite carbon. The strong diffraction at $26.3^{\circ}$ revealed the $2 \mathrm{D}$ layered structures of $\mathrm{h}-\mathrm{BCN}$ sample. The sample of h-BCN reveals an increase in the width of the reflection at $26.3^{\circ}$ and slight deviation toward smaller diffraction band, indicating the more amounts of $\mathrm{C}$ atoms within the h-BCN lattice crystals than that of h-BN, leading to an increase in the crystal lattice spacing and the formation of a new h-BCN superlattice structures. In addition, the strong diffraction peaks of (002), together with many other small diffraction peaks, suggest the 2D superlattice structures of h-BCN coexisting with a large number of lamellar stacking.

The Raman spectra for h-BCN and h-BN (Figure 4) exhibit the similar $G$ band at $1610 \mathrm{~cm}^{-1}$ and $2 \mathrm{D}$ at $2873 \mathrm{~cm}^{-1}$. The D band at $1367 \mathrm{~cm}^{-1}$ appears to be overlapped with the $G$ band. The $G$ band at $1610 \mathrm{~cm}^{-1}$ reflects the presence of graphite-like structures, symmetrical characterization, or crystallization degree $[25,30,31]$. The $2 \mathrm{D}$ band at $2873 \mathrm{~cm}^{-1}$ is associated with the graphitic-like structures. The $\mathrm{D}, \mathrm{G}$, and $2 \mathrm{D}$ bands for the h-BCN appear to be more prominent than those of h-BN, indicating the h-BCN sample has more graphitic or graphene-like structures $[32,33]$.

The chemical composition and chemical state of the samples were investigated by using XPS technique. The wide scan XPS spectra for the h-BNC in Figure 5(a) present four peaks at $196.1,291.8,409.7$, and $540.1 \mathrm{eV}$ assigned to $\mathrm{B} 1 \mathrm{~s}, \mathrm{C} 1 \mathrm{~s}, \mathrm{~N} 1 \mathrm{~s}$, and O1s, respectively [19-25]. The atomic percentage (At\%) of $\mathrm{B} 1 \mathrm{~s}, \mathrm{~N} 1 \mathrm{~s}$, and $\mathrm{C} 1 \mathrm{~s}$ was recorded as $36.1 \%, 28.4 \%$, and $20.0 \%$, respectively. The ratio of $\mathrm{B}: \mathrm{N}: \mathrm{C}$ is calculated to be about $1.8: 1.4: 1$. The $\mathrm{B} 1 \mathrm{~s}$ spectrum, as shown in Figure 5(b), was deconvoluted into two component peaks at binding energy ca. $190.4 \mathrm{eV}$ for $\mathrm{B}-\mathrm{N}$ and $191.3 \mathrm{eV}$ for B-O, respectively $[19,23]$. The N1s peak as shown in Figure 5(c) can be deconvoluted into two component peaks, namely,
N-B (398.1 eV) and N-C (pyrrolic N, $399.4 \mathrm{eV}$ ), respectively [25]. The C1 s spectrum, as shown in Figure 5(d), was deconvoluted into four component peaks at binding energy ca. $283.9 \mathrm{eV}$ for $\mathrm{C}-\mathrm{B}, 284.6 \mathrm{eV}$ for $\mathrm{C}-\mathrm{C}, 286.2 \mathrm{eV}$ for $\mathrm{C}-\mathrm{N}$, and $287.7 \mathrm{eV}$ for $\mathrm{C}-\mathrm{O}$, respectively $[23,33]$. In addition, the presence of pyrrolic $\mathrm{N}$ in five-membered rings can introduce the Faradaic reaction and thus increase the electrocatalysis performance of the electrode.

Further information about the optical absorption properties and bandgap is determined by the UV-vis absorption spectra. As can be seen by comparing h-BCN and h-BN in Figure 6(a), more carbon atoms within h-BCN represent wider range and stronger intensity of absorption. The different carbon content within h-BCN shows different behaviors of photoadsorption. The h- $\mathrm{BCN}$ displays strong absorptions at $218 \mathrm{~cm}^{-1}$ and $285 \mathrm{~cm}^{-1}$. The maximum absorption wavelength shifts toward short lengths (blue shift to $204 \mathrm{~cm}^{-1}$ and $243 \mathrm{~cm}^{-1}$, respectively) with the decrease of the amount of carbon atoms in h-BCN, indicating the doping $\mathrm{C}$ atoms enhanced the adsorption of ultraviolet and visible light capability. Figure 6(b) shows the energy level diagram of the h-BCN samples. Since the $\mathrm{BCN}$ nanosheets belong to direct bandgap semiconductor, the bandgap is measured from the plot of $(\alpha h v)^{2} v s$. $h v$ curve. The tangent line was made at the obtained curve, and the intersection point with abscissa will be the bandgap of the BCN. The bandgap for h-BCN is about $3.54 \mathrm{eV}$ and $\mathrm{h}-\mathrm{BN}$ is $3.92 \mathrm{eV}$, implying the bandgap decreased with an increase in $\mathrm{C}$ content, which is favorable for photon-generated electrons and holes under the incident light $[15,27,34]$.

3.2. Electrochemical Detection of UA on h-BCN-Modified Carbon Cloth Electrode. Figures $7(\mathrm{a})$ and $7(\mathrm{c})$ show the cyclic voltammograms of h-BCN/carbon cloth in phosphate-buffered solution ( $\mathrm{pH}$ 6.8) with the sequential microaddition of uric acid (UA) solution. For comparison, the cyclic voltammetric response for the UA on the bare carbon cloth is also shown in Figure 7(a) (inset) and 7(b). There are no characteristic redox peaks on the bare carbon cloth with the addition of UA into the electrolyte solution. The anodic current density starts near $600 \mathrm{mV}$ (vs. SCE), compared with $450 \mathrm{mV}$ on the h-BCNmodified electrode. The response current densities on the bare carbon cloth are much smaller than those of h-BCN films and do not linearly increase with the concentration of UA. Meanwhile, from Figure 6(c), the anodic peak current gradually increases linearly upon increasing concentration of UA between 10 and $500 \mu \mathrm{M}$, implying that hBCN-modified electrode behaves a high electrocatalysis activity for the UA [35]. The sensitivity of the current to concentration of UA is $0.32 \mu \mathrm{A} / \mu \mathrm{M}$ from calibration plot with correlation coefficient $\left(R^{2}\right)$ of 0.991 .

3.3. Electrochemical Detection of DA on h-BCN-Modified Carbon Cloth Electrode. The electrochemical behavior for $\mathrm{DA}$ at h-BCN/carbon cloth and bare carbon cloth was studied by CV. It can be seen from Figure 8(a) that the current responses at DA on carbon cloth start at about $550 \mathrm{mV}$, but 


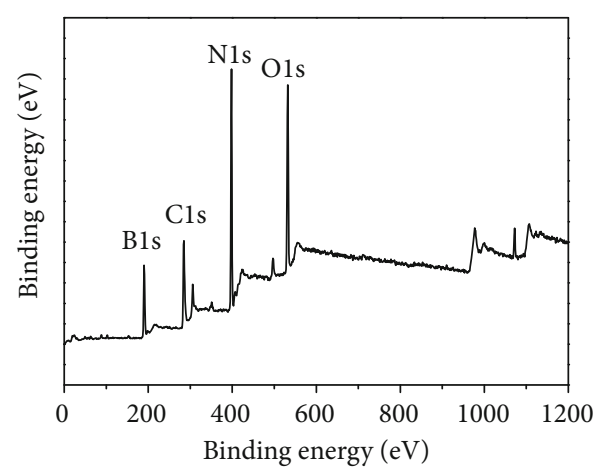

(a)

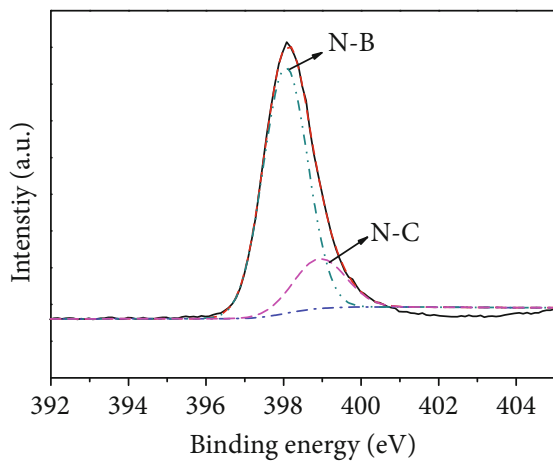

(c)

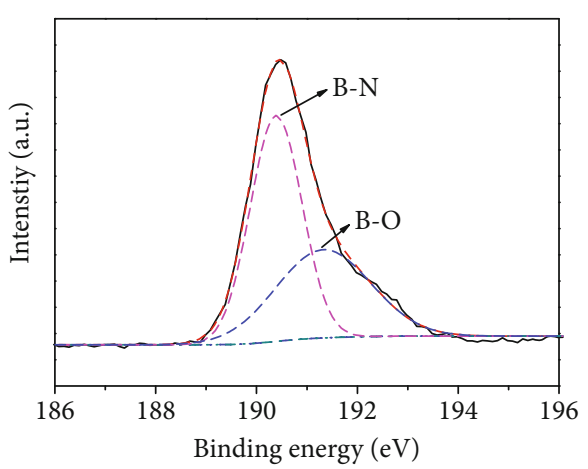

(b)

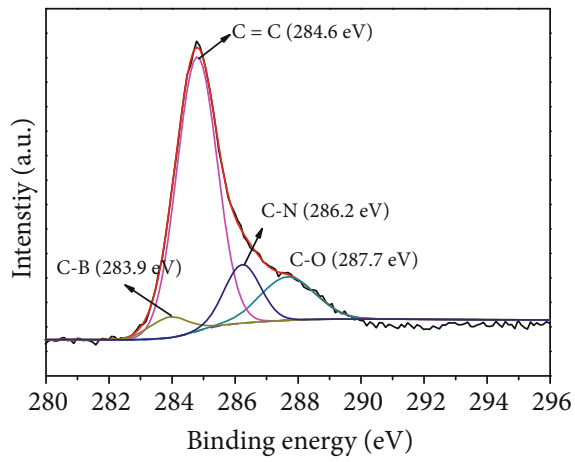

(d)

Figure 5: (a) XPS survey spectrum of h-BCN. (b, c, and d) High-resolution XPS spectra of B1s (b), N1s (c), and C1s (d).

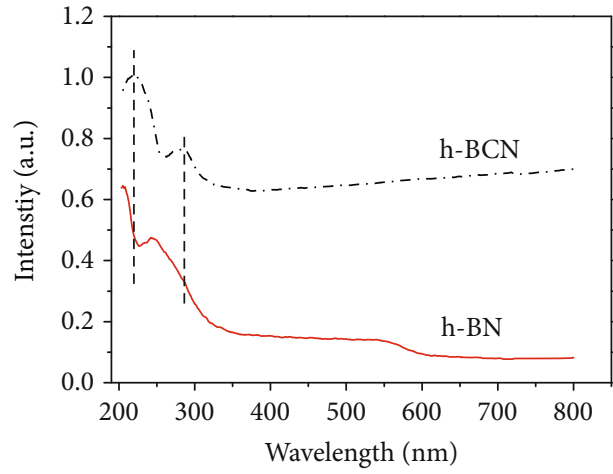

(a)

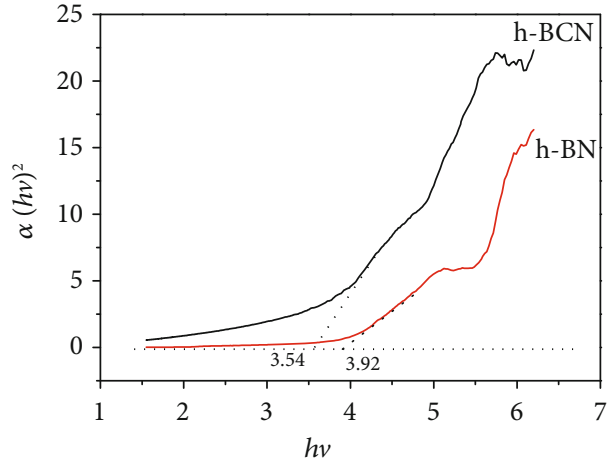

(b)

FIgURE 6: (a) UV spectrometer of h-BCN and h-BN. (b) Energy level diagram of the h-BCN and h-BN samples.

there is no linear relationship between current densities and concentration of DA, suggesting that the bare carbon cloth cannot be used as a sensor for DA. In the case of h-BCNmodified film in Figure $8(\mathrm{~b})$, the anodic peak current at $650 \mathrm{mV}$ vs. SCE increases linearly upon increasing concentration of DA between 10 and $700 \mu \mathrm{M}$. The current responses for DA at h-BCN/carbon cloth are much larger than those of bare carbon cloth. The sensitivity of the current to concentration of DA is $0.688 \mu \mathrm{A} / \mu \mathrm{M}$ from calibration plot with correlation coefficient $\left(R^{2}\right)$ of 0.992 which is an indication of a good linear relationship.
Amperometric technique was also used to determine the DA content in the phosphate-buffered solution $(\mathrm{pH}$ 6.86). Figure 8(d) shows the amperometric responses of the h-BCN-modified carbon cloth to sequential microadditions of DA at $650 \mathrm{~m} \mathrm{~V}$. From the inset calibration plot, a sensitivity of $0.24 \mu \mathrm{A} / \mu \mathrm{M}$ with correlation coefficient $\left(R^{2}\right)$ of 0.97 was obtained, which is comparable to other electrochemical sensors for DA $[36,37]$. This high level of the sensitivity is mostly due to the electrocatalytic activities from $\mathrm{h}-\mathrm{BCN}$ semiconductor. The detection limit $(S / N=3)$ was evaluated to be $5 \mu \mathrm{M}$. The repeatability of an electrode 

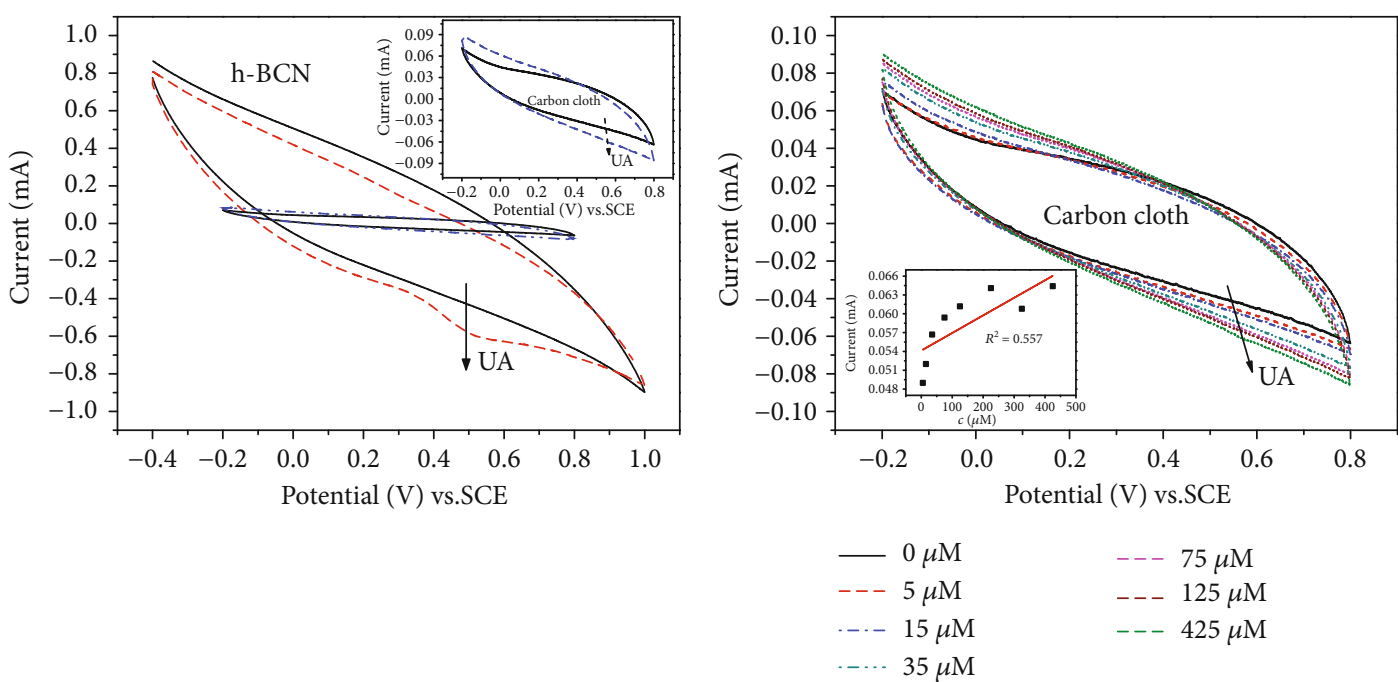

(a)

(b)

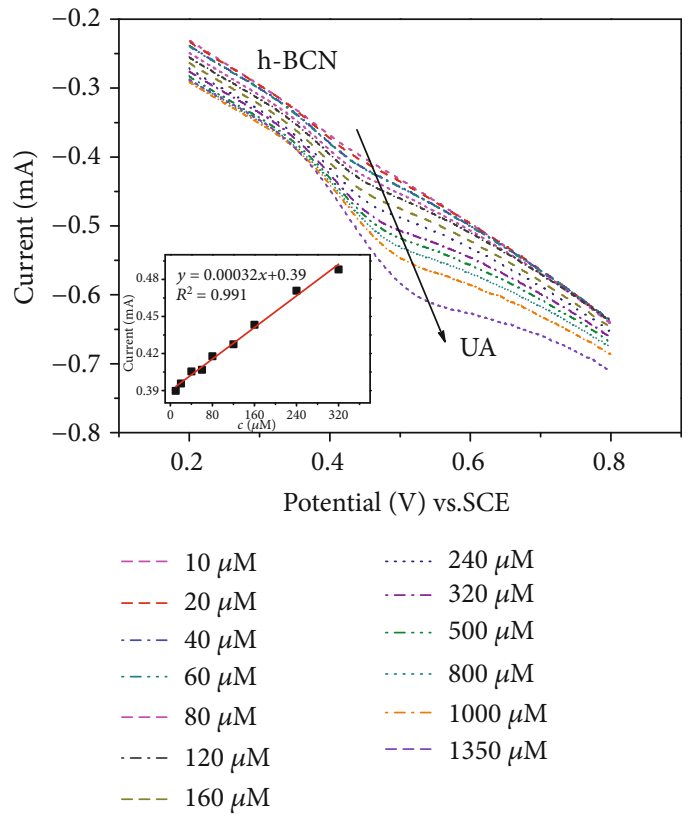

(c)

Figure 7: (a) CV curve of h-BCN-modified electrode and bare carbon cloth electrodes (inset highlighted) in phosphate-buffered solution (pH 6.8) and in buffer solution containing $200 \mu \mathrm{M}$ UA. (b) CV curves of bare carbon cloth at various UA concentrations (5-425 $\mu \mathrm{M})$. Inset shows the calibration plot for UA concentration. (c) $\mathrm{CV}$ curves of h-BCN-modified electrode at various UA concentrations (10-500 $\mu \mathrm{M})$ in phosphate-buffered solution ( $\mathrm{pH}$ 6.8). Inset shows the calibration plot for UA concentration. Scan rate, $10 \mathrm{mVs}^{-1}$.

evaluated by three repetitive measurements of $20 \mu \mathrm{M} \mathrm{DA}$ gave an R.S.D. of $3.1 \%$. The reproducibility of three composite electrodes evaluated similarly gave an R.S.D. of $4.9 \%$. These experiment data show the h-BCN-modified electrode is a promise candidate for application in detection of DA and UA.

\section{Conclusion}

Interlayered hybrid h-BCN was successfully synthesized by in situ solid heterogeneous reaction subsequently with reduction in the hydrazine solution using anhydrous borax, melamine, and the low molecular weight phenolic resin as starting materials. TEM and XRD measurements revealed the two different interlayer lattice fringes of 0.34 and $0.21 \mathrm{~nm}$ presented within the $\mathrm{h}-\mathrm{BCN}$, indicating the $2 \mathrm{D}$ superlattice interlayered structures of h-BCN. UV-vis absorption measurement demonstrated the doping $\mathrm{C}$ atoms in the h-BN enhanced the adsorption of ultraviolet and visible light capability. The bandgap of $\mathrm{h}-\mathrm{BCN}$ is smaller than that of h-BN, which is beneficial for the semiconductor nature of h-BCN. h-BCN represents 


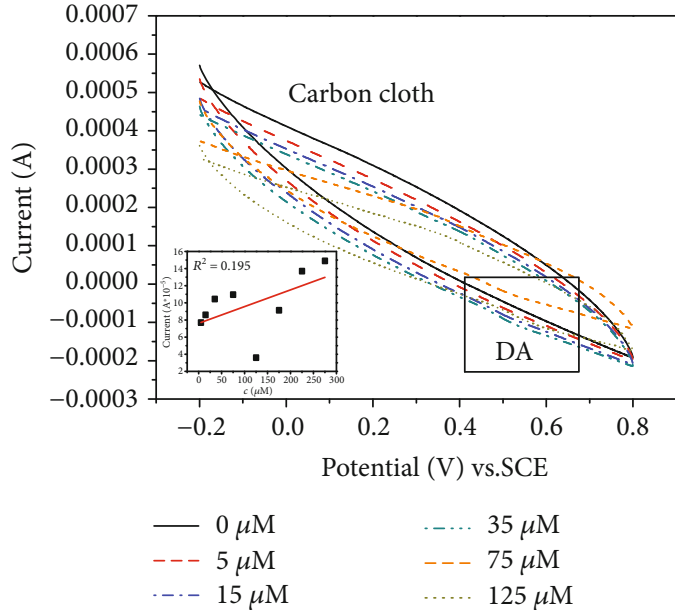

(a)

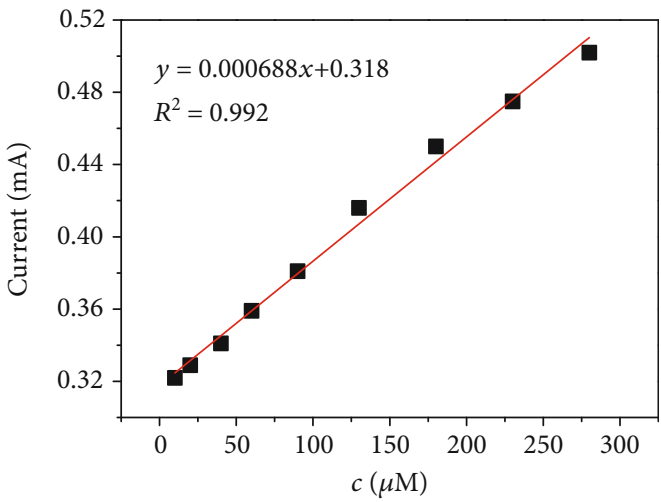

(c)

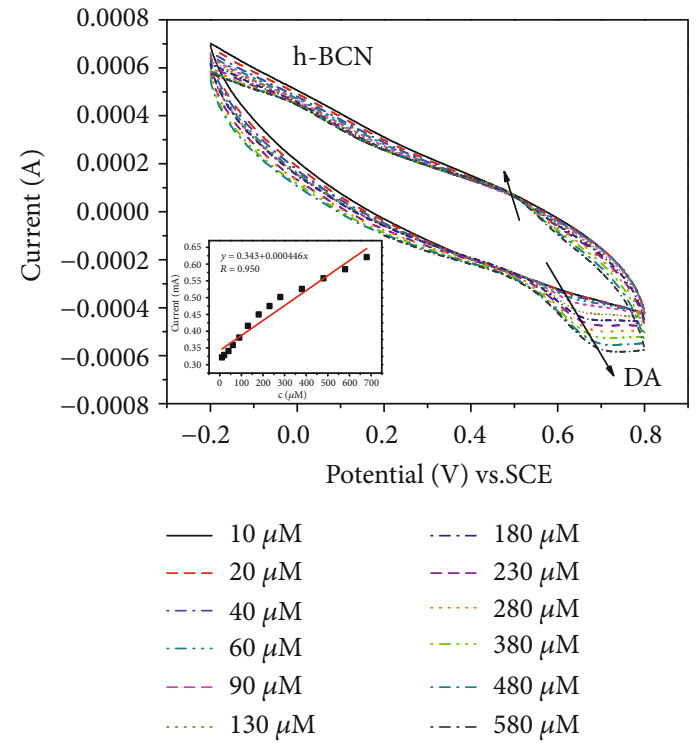

(b)

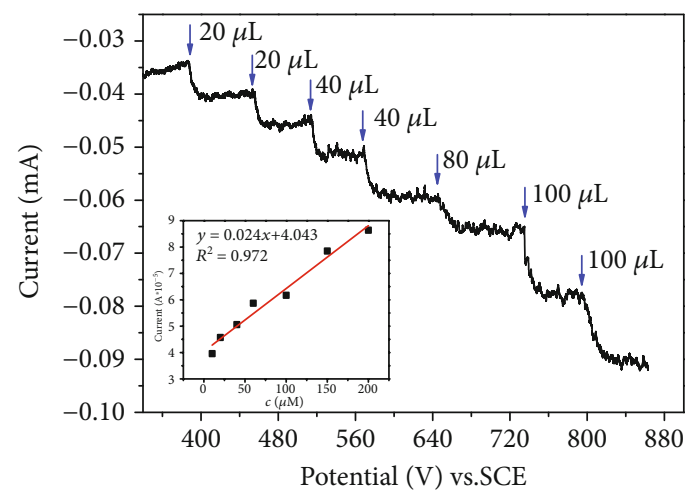

(d)

FIgURE 8: (a) CV curves of bare carbon cloth at various UA concentrations in phosphate-buffered solution (10-200 $\mu \mathrm{M})$. Inset shows the calibration plot for UA concentration. (b) Cyclic voltammograms of h-BCN-modified electrode at various DA concentrations (10-580 $\mu \mathrm{M}$ ) in phosphate-buffered solution ( $\mathrm{pH}$ 6.8). Inset shows the calibration plot for UA concentration. Scan rate, $10 \mathrm{mVs}^{-1}$. (c) The calibration plot for UA concentration from 10 to $300 \mu \mathrm{M}$. (d) Amperometric response of h-BCN-modified electrode at $0.65 \mathrm{~V}$ for the successive microadditions of DA to buffer solution (corresponding to $10-200 \mu \mathrm{M}$ ). Inset shows the calibration plot for DA concentration.

excellent electrocatalysis performance for uric acid and dopamine with high level of the sensitivities and low detection limits, indicating a good candidate for electrochemical sensor applications.

\section{Data Availability}

The data used to support the findings of this study are included within the article.

\section{Conflicts of Interest}

The authors declare that they have no conflicts of interest.

\section{Acknowledgments}

We acknowledge Liaoning Key Laboratory of Functional Textile Materials and Dalian Institute of Chemical Physics for the technical support of SEM, TEM, and BET measurements. We acknowledge the financial support by the National Natural Science Foundation of China (project number 51343002).

\section{References}

[1] F. Li, J. Gao, J. Zhang, F. Xu, J. Zhao, and L. Sun, "Graphene oxide and lithium amidoborane: a new way to bridge chemical and physical approaches for hydrogen storage," Journal of Materials Chemistry A, vol. 1, no. 27, pp. 8016-8022, 2013.

[2] C. Liu, Z. Yu, D. Neff, A. Zhamu, and B. Z. Jang, "Graphenebased supercapacitor with an ultrahigh energy density," Nano Letters, vol. 10, no. 12, pp. 4863-4868, 2010.

[3] F. Xiao, J. Song, H. Gao, X. Zan, R. Xu, and H. Duan, "Coating graphene paper with $2 \mathrm{D}$-assembly of electrocatalytic nanoparticles: a modular approach toward high-performance flexible electrodes," ACS Nano, vol. 6, no. 1, pp. 100-110, 2012. 
[4] A. A. Balandin, S. Ghosh, W. Bao et al., "Superior thermal conductivity of single-layer graphene," Nano Letters, vol. 8, no. 3, pp. 902-907, 2008.

[5] C. N. R. Rao, H. S. S. Ramakrishna Matte, and U. Maitra, "Graphene analogues of inorganic layered materials," Angewandte Chemie International Edition, vol. 52, no. 50, pp. 1316213185, 2013.

[6] H.-B. Cho, Y. Tokoi, S. Tanaka et al., "Modification of BN nanosheets and their thermal conducting properties in nanocomposite film with polysiloxane according to the orientation of BN," Composites Science and Technology, vol. 71, no. 8, pp. 1046-1052, 2011.

[7] R. N. Muthu, S. Rajashabala, and R. Kannan, "Hexagonal boron nitride (h-BN) nanoparticles decorated multi-walled carbon nanotubes (MWCNT) for hydrogen storage," Renewable Energy, vol. 85, pp. 387-394, 2016.

[8] J. Hu, Y. Huang, X. Zeng et al., "Polymer composite with enhanced thermal conductivity and mechanical strength through orientation manipulating of BN," Composites Science and Technology, vol. 160, pp. 127-137, 2018.

[9] J. Li, J. Lin, X. Xu et al., "Porous boron nitride with a high surface area: hydrogen storage and water treatment," Nanotechnology, vol. 24, no. 15, pp. 155-603, 2013.

[10] W. Cai, W. Guo, Y. Pan et al., "Polydopamine-bridged synthesis of ternary h-BN@PDA@SnO ${ }_{2}$ as nanoenhancers for flame retardant and smoke suppression of epoxy composites," Composites Part A: Applied Science and Manufacturing, vol. 111, pp. 94-105, 2018.

[11] F. Mahvash, E. Paradis, D. Drouin, T. Szkopek, and M. Siaj, "Space-charge limited transport in large-area monolayer hexagonal boron nitride," Nano, vol. 15, no. 4, pp. 2263-2268, 2015.

[12] C. Zhi, Y. Bando, C. Tang, H. Kuwahara, and D. Golberg, "Large-scale fabrication of boron nitride nanosheets and their utilization in polymeric composites with improved thermal and mechanical properties," Advanced Materials, vol. 21, no. 28, pp. 2889-2893, 2009.

[13] Z. Su, H. Wang, X. Ye et al., "Synergistic enhancement of anisotropic thermal transport flexible polymer composites filled with multi-layer graphene $(\mathrm{mG})$ and mussel-inspiring modified hexagonal boron nitride (h-BN)," Composites Part A: Applied Science and Manufacturing, vol. 111, pp. 12-22, 2018.

[14] J. Wang, F. Ma, W. Liang, R. Wang, and M. Sun, "Optical, photonic and optoelectronic properties of graphene, h-BN and their hybrid materials," Nano, vol. 6, no. 5, pp. 943-976, 2017.

[15] J. Wang, F. Ma, W. Liang, and M. Sun, "Electrical properties and applications of graphene, hexagonal boron nitride (h$\mathrm{BN})$, and graphene/h-BN heterostructures," Materials Today Physics, vol. 2, pp. 6-34, 2017.

[16] Q. Li, C. Huo, K. Yi, L. Zhou, L. Su, and X. Hou, "Preparation of flake hexagonal $\mathrm{BN}$ and its application in electrochemical detection of ascorbic acid, dopamine and uric acid," Sensors and Actuators B, vol. 260, pp. 346-356, 2018.

[17] J. Wang, F. Ma, and M. Sun, "Graphene, hexagonal boron nitride, and their heterostructures: properties and applications," RSC Advances, vol. 7, no. 27, pp. 16801-16822, 2017.

[18] S. Beniwal, J. Hooper, D. P. Miller et al., "Graphene-like boron -carbon-nitrogen monolayers," ACS Nano, vol. 11, no. 3, pp. 2486-2493, 2017.
[19] S. Saha, P. Samanta, N. C. Murmu et al., "Modified electrochemical charge storage properties of h-BN/rGO superlattice through the transition from $\mathrm{n}$ to $\mathrm{p}$ type semiconductor by fluorine doping," Chemical Engineering Journal, vol. 339, pp. 334$345,2018$.

[20] G. H. Han, J. A. Rodríguez-Manzo, C. W. Lee et al., "Continuous growth of hexagonal graphene and boron nitride in-plane heterostructures by atmospheric pressure chemical vapor deposition," ACS Nano, vol. 7, no. 11, pp. 10129-10138, 2013.

[21] D. Kim, A. Hashmi, C. Hwang, and J. Hong, "Thickness dependent band gap and effective mass of BN/graphene/BN and graphene/BN/graphene heterostructures," Surface Science, vol. 610, pp. 27-32, 2013.

[22] Q. Wu, W. Wongwiriyapan, J. H. Park et al., "In situ chemical vapor deposition of graphene and hexagonal boron nitride heterostructures," Current Applied Physics, vol. 16, no. 9, pp. 1175-1191, 2016.

[23] L. Ci, L. Song, C. Jin et al., "Atomic layers of hybridized boron nitride and graphene domains," Nature Materials, vol. 9, no. 5, pp. 430-435, 2010.

[24] R. Chegel, "Bias induced modulation of electrical and thermal conductivity and heat capacity of BN and BN/graphene bilayers," Physica B: Condensed Matter, vol. 511, pp. 26-35, 2017.

[25] S. Saha, P. Samanta, N. C. Murmu, N. H. Kim, T. Kuila, and J. H. Lee, "Electrochemical functionalization and in-situ deposition of the SAA@rGO/h-BN@Ni electrode for supercapacitor applications," Journal of Industrial and Engineering Chemistry, vol. 52, pp. 321-330, 2017.

[26] T. P. Kaloni, Y. C. Cheng, and U. Schwingenschlogl, "Electronic structure of superlattices of graphene and hexagonal boron nitride," Journal of Materials Chemistry, vol. 22, no. 3, pp. 919-922, 2012.

[27] S. Tang, J. Yu, and L. Liu, "Tunable doping and band gap of graphene on functionalized hexagonal boron nitride with hydrogen and fluorine," Physical Chemistry Chemical Physics, vol. 15, no. 14, pp. 5067-5077, 2013.

[28] J. Wang, X. Mu, X. Wang et al., "The thermal and thermoelectric properties of in-plane $\mathrm{C}-\mathrm{BN}$ hybrid structures and graphene/h-BN van der Waals heterostructures," Materials Today Physics, vol. 5, pp. 29-57, 2018.

[29] J. Wang, X. Xu, X. Mu, F. Ma, and M. Sun, "Magnetics and spintronics on two-dimensional composite materials of graphene/hexagonal boron nitride," Materials Today Physics, vol. 3, pp. 93-117, 2017.

[30] L. Song, L. Ci, H. Lu et al., "Large scale growth and characterization of atomic hexagonal boron nitride layers," Nano Letters, vol. 10, no. 8, pp. 3209-3215, 2010.

[31] M. P. Levendorf, C. J. Kim, L. Brown et al., "Graphene and boron nitride lateral heterostructures for atomically thin circuitry," Nature, vol. 488, no. 7413, pp. 627-632, 2012.

[32] Z. Zhang and W. Guo, "Controlling the functionalizations of hexagonal boron nitride structures by carrier doping," Journal of Physical Chemistry Letters, vol. 2, no. 17, pp. 2168-2173, 2011.

[33] B.-X. Zou, Y. Gao, B. Liu, Y. Yu, and Y. Lu, “Three dimensional heteroatom-doped carbon composite film for flexible solidstate supercapacitors," RSC Advances, vol. 6, no. 6, pp. 44834489, 2016.

[34] Z. Liu, L. Ma, G. Shi et al., "In-plane heterostructures of graphene and hexagonal boron nitride with controlled domain sizes," Nature Nanotech, vol. 8, no. 2, pp. 119-124, 2013. 
[35] B.-X. Zou, X.-X. Liu, D. Diamond, and K.-T. Lau, "Electrochemical synthesis of $\mathrm{WO}_{3} / \mathrm{PANI}$ composite for electrocatalytic reduction of iodate," Electrochimica Acta, vol. 55, no. 12, pp. 3915-3920, 2010.

[36] X. Lin, Q. Zhuang, J. Chen, S. Zhang, and Y. Zheng, "Electrocatalytic property of poly-chromotrope $2 \mathrm{~B}$ modified glassy carbon electrode on dopamine and its application," Sensors and Actuators B, vol. 125, no. 1, pp. 240-245, 2007.

[37] A. Balamurugan and S.-M. Chen, "Poly(3,4-ethylenedioxythiophene-co-(5-amino-2-naphthalenesulfonic acid)) (PEDOTPANS) film modified glassy carbon electrode for selective detection of dopamine in the presence of ascorbic acid and uric acid," Analytica Chimica Acta, vol. 596, no. 1, pp. 92-98, 2007. 


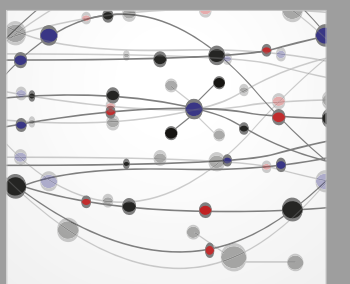

The Scientific World Journal
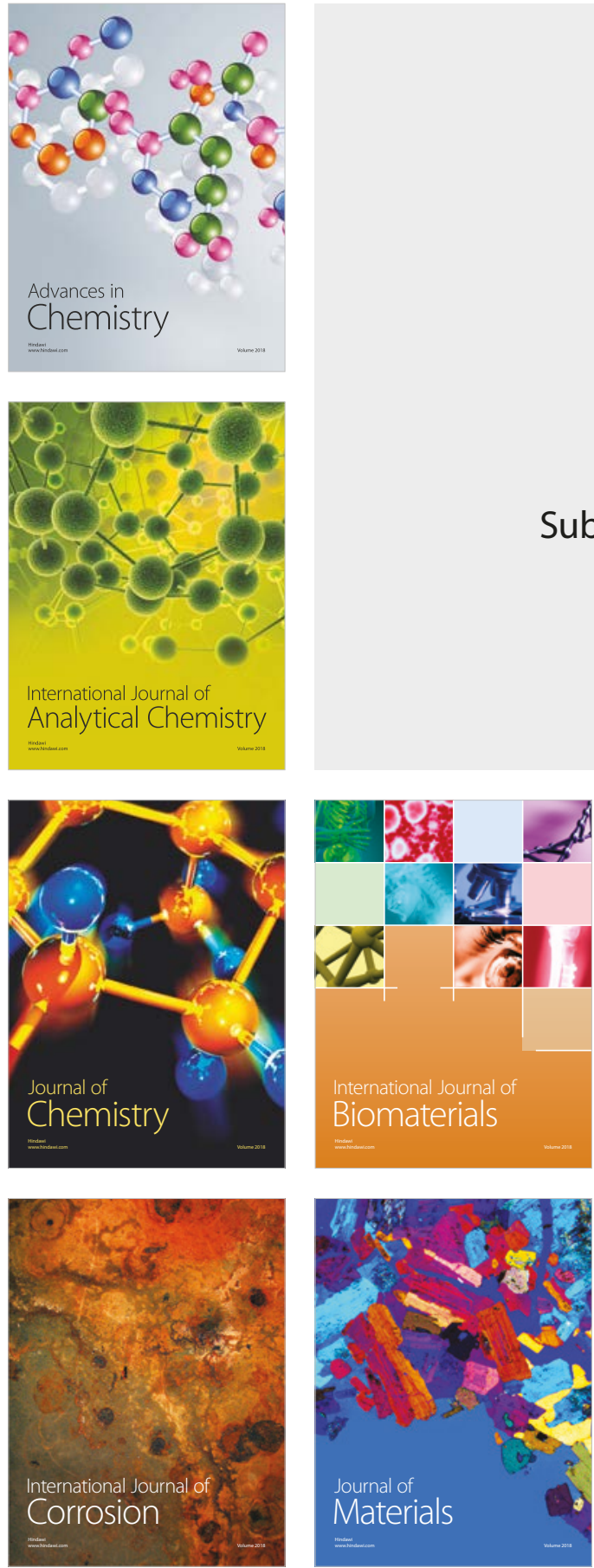

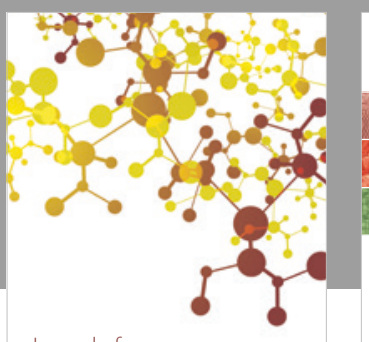

Journal of

Applied Chemistry
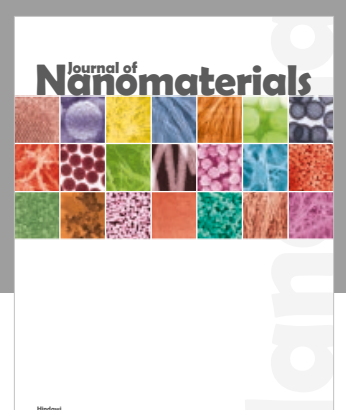

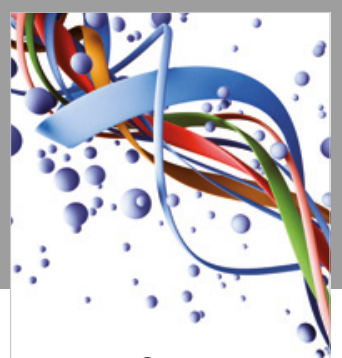

Scientifica

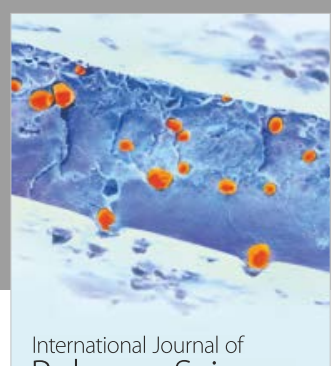

Polymer Science

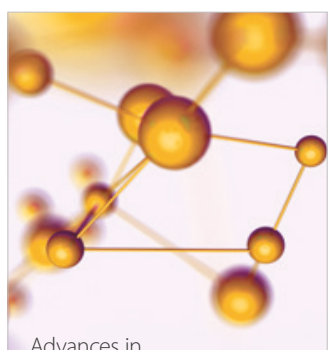

Physical Chemistry
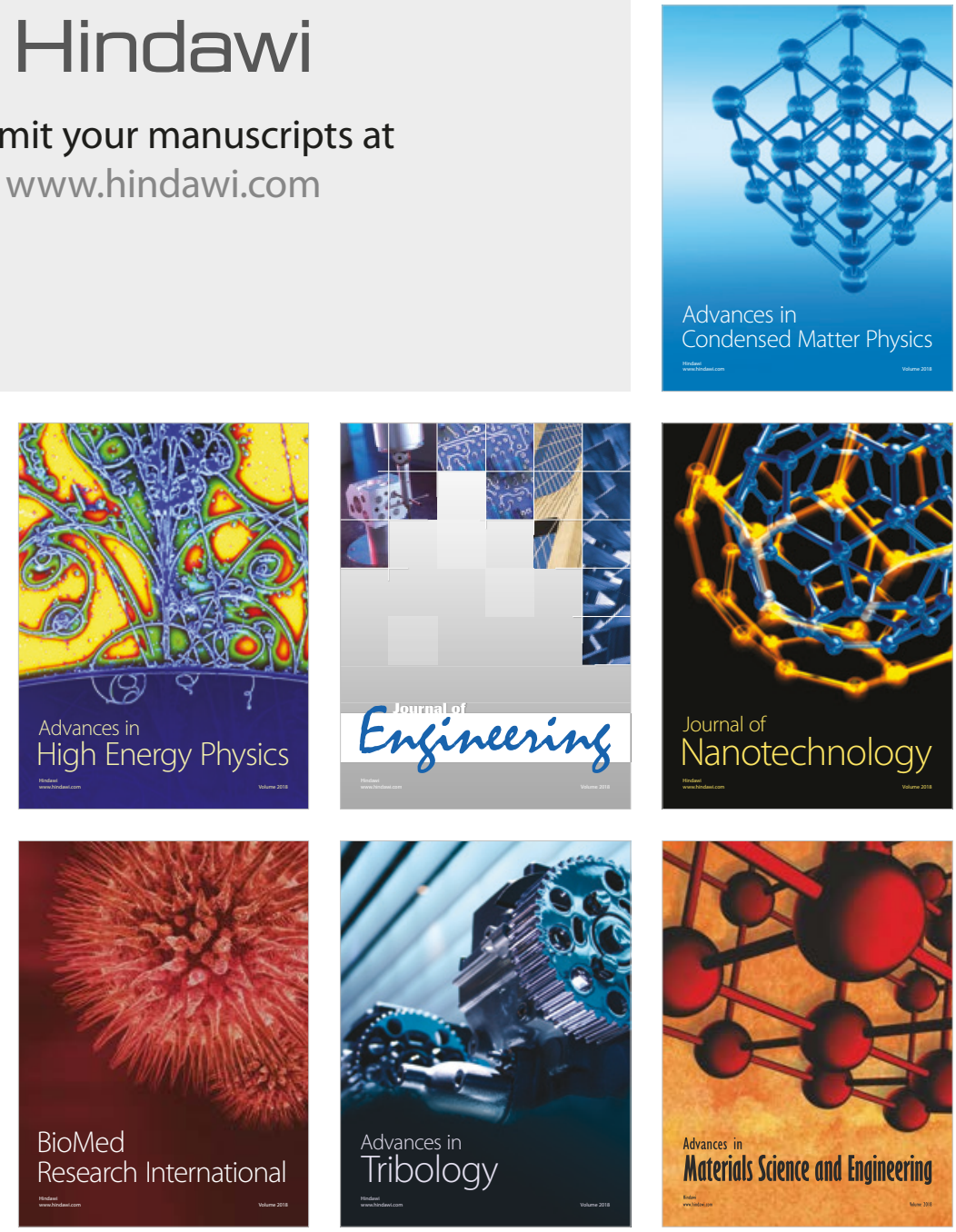